\title{
Elastic instability in the thermocapillary convection at low Reynolds numbers
}

\author{
Kai-Xin $\mathrm{Hu}^{\mathrm{a}, *}$, Rong Liu ${ }^{\mathrm{b}}$, Meng $\mathrm{He}^{\mathrm{c}}$, Qi-Sheng Chen ${ }^{\mathrm{c}}$ \\ a School of Mechanical Engineering and Mechanics, Ningbo University, Ningbo, Zhejiang 315211, China \\ ${ }^{\mathrm{b}}$ School of Mechanical and Electrical Engineering, Gui Lin University of Electronic Technology, Gui Lin 541004, China \\ ${ }^{\mathrm{c}}$ National Microgravity Laboratory, Institute of Mechanics, Chinese Academy of Sciences, Beijing 100190, China
}

\section{A R T I C L E I N F O}

\section{Keywords:}

Viscoelastic

Elastic instability

Thermocapillary

\begin{abstract}
A B S T R A C T
It is well known that the plane channel flows of UCM and Oldroyd-B fluids are linearly stable at low Reynolds numbers. However, an elastic instability is detected by modal analysis in the thermocapillary convection with straight streamlines. The instability appears when the Weissenberg number is in the order of 1 . When the Reynolds number is small enough, the critical Weissenberg number is almost a constant, which only depends on the Marangoni number. The unstable waves include a wide range of wave number and can be streamwise and oblique. The free surface with thermocapillary effect is crucial for the instability, as it induces the normal stress in the basic flow and reduces the constraint on perturbations.
\end{abstract}

\section{Introduction}

The flow instabilities of viscoelastic fluids are of great practical importance for polymer processing operations. The study for these phenomena has lasted over the years [1]. In many applications such as the extrusion of polymer melt, the instability occurs at low Reynolds numbers, which is quite different from the hydrodynamic instability at high Reynolds numbers of Newtonian fluid. In order to identify its mechanism, many studies have been devoted to the stability of shear flow of viscoelastic fluids. A purely elastic instability has been discovered in Taylor-Couette flow by linear stability analysis [2], where the curved streamlines are crucial for the instability mechanism [3]. In contrast, the parallel shear flows of viscoelastic fluids in channels are always linearly stable at low Reynolds numbers $[4,5]$. The viscoelastic channel flows are highly susceptible to non-modal amplification of disturbances [6-10], and the elastic instabilities reported are associated with nonlinearity [11]. However, in this paper, an elastic instability is detected by linear stability analysis in the thermocapillary convection at low Reynolds numbers.

Thermocapillary convection refers to the shear flow driven by the temperature-induced surface tension gradient. It has been an active research topic for its practical application in the crystal growth techniques [12]. The thermocapillary flows in polymer liquids are known to occur in many industrial applications, such as film drying [13], optical lithography [14], deliberate patterning of polymer [15], inkjet printing [16] and polymer processing in microgravity [17]. It is worth noting that the polymer liquids are often viscoelastic fluids, whose rheological properties differ significantly from Newtonian fluids. The effect of elasticity should be considered in the flow of polymeric fluid.

A few investigations have been undertaken to some problems of viscoelastic thermocapillary convections. Corbett \& Kumar [18] have examined the thermocapillary patterning of polymer films via a linear stability analysis and nonlinear simulations. The Jeffreys model is used to investigate viscoelastic effects. It suggests that the thermal and electrohydrodynamic effects can be used simultaneously in the patterning. Kaloni \& Lou [19] have demonstrated the influence of thermocapillary forces on the stability of buoyancy-driven viscoelastic fluid layer. But they only considered the cases when the buoyancy force is much larger than the thermocapillary force with Prandtl numbers 10 and 100. Davalos-Orozco \& Chavez [20] have investigated analytically the two-dimensional linear thermocapillary instability of a thin viscoelastic fluid layer under a horizontal temperature gradient. The solution obtained is based on the small wavenumber approximation. The instability of thermocapillary liquid layers for Oldroyd-B fluid has been studied by Hu et al. [21] with Prandtl numbers 0.02 and 100. Three kinds of instabilities are found in different elasticity, which includes oblique wave, streamwise wave and spanwise stationary mode. The flow is stabilized at small elasticity but destabilized at high elasticity. However, the parameters examined in the above works are limited. There are still many problems remaining. For example, the stability of thermocapillary convection at low Reynolds number and high elastic number (the ratio of Weissenberg number to Reynolds number) has not

\footnotetext{
" Corresponding author.

E-mail address: hjhhkx@126.com (K.X. Hu).
} 
been investigated, although this case appears in many flows of polymer liquid. Thus, it is natural to ask whether a pure elastic instability can exist in the thermocapillary convection at low Reynolds number, which is the purpose of this paper.

In the present work, the linear stability of viscoelastic thermocapillary convection is investigated at low Reynolds numbers. The model of thermocapillary liquid layers is considered, while the rheological property of polymer liquid is modeled by the Oldroyd-B fluid. The flow state is susceptible to an elastic instability when the Weissenberg number is in the order of 1 . The critical parameters are determined.

This paper is organized as follows. In Section 2, the equations of the viscoelastic thermocapillary convection are formulated. The solution of the basic flow and the dimensionless governing equations are derived. Then in Section 3, the properties of elastic instability are demonstrated. The wave speed and growth rate for the perturbation are displayed. The critical parameters for the instability are obtained, and the energy mechanism is studied. After that, the comparisons are made with other instabilities of viscoelastic fluids in Section 4. Finally, the conclusions are drawn in Section 5.

\section{Problem formulation}

In the theoretical study of thermocapillary convections, the thermocapillary liquid layer presented by Smith and Davis [22] has been widely used, where the fluid on an infinite rigid plane is set in motion by the temperature gradient on the free surface (see Fig. 1). The oblique hydrothermal wave predicted by this model has been observed in both experiment [23] and numerical simulation [24]. The model includes two kinds of plane shear flows, the linear flow and return flow. The former has homogeneous velocity gradient, while the latter is a sum of plane Couette and Poiseuille flow with zero mass flux through the vertical section. In Fig. $1, d$ is the depth of the layer, $u_{0}$ is the velocity, $x$ is the streamwise direction, $z$ is the wall-normal direction, and $y$ is the spanwise direction. For simplicity, we suppose the surface tension is big enough that the deformation of free surface is not considered as that in

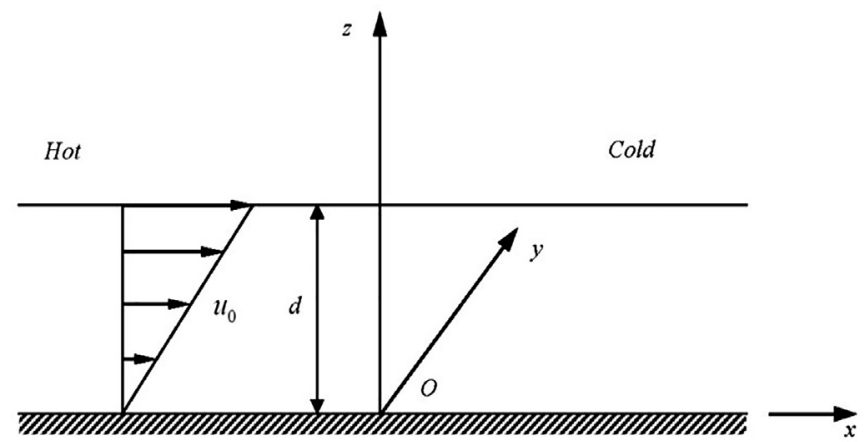

(a)

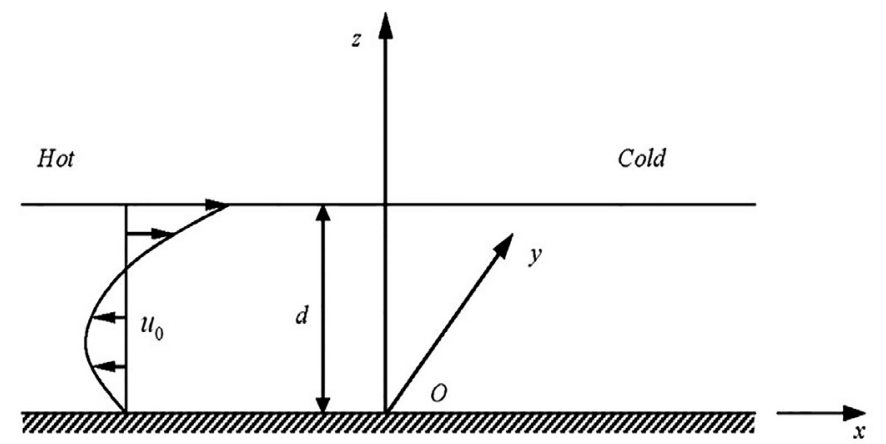

(b)

Fig. 1. The model of thermocapillary liquid layers: (a) linear flow and (b) return flow.
[22]. This assumption is satisfied in some applications, such as liquid silicon [22] and silicone oil [23].

\subsection{Governing equations}

In our work, $R$ is defined as $R=\frac{\rho U d}{\mu}$, where $\rho, \mu$ are the fluid density and viscosity, respectively. $U$ is the characteristic velocity defined as $U=b \gamma d / \mu, b$ is the temperature gradient on the surface and $\gamma$ is the negative rate of change of surface tension with the temperature. The Marangoni number is defined as $M a=b \gamma d^{2} / \mu \chi$, where $\chi$ is the thermal diffusivity. There is a relation between $M a$ and $R M a=R \cdot P r$, where $P r$ is the Prandtl number. These definitions are the same as those of Newtonian fluid in [22].

The dimensionless governing equations of the flow are as follows, which are the continuity equation, momentum equation and energy equation, respectively.

$\nabla \cdot \mathbf{u}=0$

$R\left(\frac{\partial \mathbf{u}}{\partial t}+\mathbf{u} \cdot \nabla \mathbf{u}\right)=-\nabla p+\nabla \cdot \mathbf{Q}$,

$\frac{\partial T}{\partial t}+\mathbf{u} \cdot \nabla T=\frac{1}{M a} \nabla^{2} T$.

Here $\mathbf{u}, p, T$ are the velocity field, pressure and temperature, respectively. $\mathbf{Q}$ is the stress tensor. The heat produced by viscous dissipation is ignored in the right side of (2.3).

We use the Oldroyd-B fluid to model the rheological property of the polymer liquid as it has been widely used in the theoretical studies $[1,2]$. Its dimensionless constitutive equation is [21]

$\left(1+\lambda \frac{\delta}{\delta t}\right) \mathbf{Q}=\left(1+\lambda \widetilde{\beta} \frac{\delta}{\delta t}\right) \mathbf{S}$.

Here $\mathbf{S}$ is the strain-rate tensor,

$\mathbf{S}=\nabla \mathbf{u}+(\nabla \mathbf{u})^{\mathrm{T}}$,

$\frac{\delta}{\delta t}$ is the upper convected derivative with the expression of

$\frac{\delta \mathbf{Q}}{\delta t}=\frac{\partial \mathbf{Q}}{\partial t}+\mathbf{u} \cdot \nabla \mathbf{Q}-(\nabla \mathbf{u})^{\mathrm{T}} \cdot \mathbf{Q}-\mathbf{Q} \cdot(\nabla \mathbf{u})$.

$\lambda$ is the Weissenberg number, which has $\lambda=\frac{\mu}{G} \frac{U}{d}, G$ is the elastic modulus. $\widetilde{\beta}$ is the ratio of solvent viscosity to the total viscosity. The Oldroyd-B fluid recovers a Newtonian fluid when $\lambda=0$.

Although the upper-convected Maxwell (UCM) fluid is simpler (It is a special case of Oldroyd-B fluid when $\widetilde{\beta}=0$.), and has been applied in many previous works of polymer melt $[1,5,25]$, we cannot use it in the present work for the following reason. This model has an instantaneous elasticity, which often gives rise to short-wave instabilities with unbounded growth rates [26]. We find that the short-wave instability also appears in the thermocapillary convection of UCM fluid when the Weissenberg number is unrealistically low, which indicates that the UCM fluid model needs to be modified for the problem in this paper. Joseph \& Saut [26] suggested that this phenomenon can be regularized by using the Oldroyd-B fluid, which has an additional Newtonian viscosity to the UCM fluid. Therefore, we use the Oldroyd-B fluid in the following and set $\widetilde{\beta}=0.001$, which means that the Newtonian viscosity is significantly lesser. The reason why we chose this value is that, the flow is stabilized by the increase of $\widetilde{\beta}$, and the critical parameters determined by our computation can be comparable with the some fluid parameters with $\widetilde{\beta}=0.001$.

The boundary conditions are set as follows. On the wall, there is no slip and zero heat flux:

$\mathbf{u}=(u, v, w)=0, \frac{\partial T}{\partial z}=0, z=0$,

on the free surface, 
$Q_{13}+\frac{\partial T}{\partial x}=0, Q_{23}+\frac{\partial T}{\partial y}=0, w=0,-\frac{\partial T}{\partial z}=B i \cdot\left(T-T_{\infty}\right)+\widetilde{Q}, z=1$.

Here, the first two equations stand for the relation between the temperature gradient and stress caused by thermocapillary effect. $T_{\infty}$ is the temperature of the bounding gas far from the surface.Bi is the Biot number. $\widetilde{Q}$ is the imposed heat flux to the environment, which is not an independent parameter [22]. In the experiment, $\widetilde{Q}$ is determined by setting a horizontal temperature gradient in the liquid $\left(\frac{\partial T}{\partial x}=-1\right)$. Then a parallel shear flow $u=u(z)$ is induced by the thermocapillary effect, and a temperature distribution in $z$ is obtained from a balance between the vertical conduction and horizontal convection: $\frac{1}{M a} \frac{\partial^{2} T}{\partial z^{2}}=-u(z)$. So $\left.\frac{\partial T}{\partial z}\right|_{z=1}$ can be deduced by the velocity field $u(z)$ and the condition $\left.\frac{\partial T}{\partial z}\right|_{z=0}$ as follows: $\left.\frac{\partial T}{\partial z}\right|_{z=1}=\left.\frac{\partial T}{\partial z}\right|_{z=0}+\int_{0}^{1} \frac{\partial^{2} T}{\partial z^{2}} \mathrm{~d} z=-M a \int_{0}^{1} u(z) \mathrm{d} z \cdot T_{\infty}$ is passive and depends on the temperature of liquid surface. Then, $\widetilde{Q}$ is introduced for the energy balance on the surface and can be determined by the particular basic-state solution as follows [22]:

$\widetilde{Q}=-\left.\frac{\partial T}{\partial z}\right|_{z=1}-B i \cdot\left(\left.T\right|_{z=1}-T_{\infty}\right)$.

The solutions of the basic flow are derived as follows. The velocity field is a linear combination of plane Couette and Poiseuille flow, while the temperature field is linear in $x$ as imposed plus a distribution in $z$ :

$u_{0}(z)=u_{0}^{\prime}(1) z+\frac{1}{2} u_{0}^{\prime \prime}(1)\left(z^{2}-2 z\right), v_{0}=w_{0}=0$,

$$
\begin{aligned}
T_{0}(x, z)= & -u_{0}^{\prime}(1) x+M a \cdot u_{0}^{\prime}(1)\left\{\frac{1}{24} u^{\prime^{\prime}}{ }_{0}(1)\left(1-z^{4}\right)\right. \\
& \left.+\frac{1}{6}\left[u_{0}^{\prime}(1)-u^{\prime^{\prime}}{ }_{0}(1)\right]\left(1-z^{3}\right)\right\} .
\end{aligned}
$$

These solutions are the same as those for Newtonian fluid in [22]. The strain-rate and stress tensor of the basic flow have the following form:

$\mathbf{S}_{0}=u_{0}^{\prime}(z)\left[\begin{array}{lll}0 & 0 & 1 \\ 0 & 0 & 0 \\ 1 & 0 & 0\end{array}\right], \mathbf{Q}_{0}=u_{0}^{\prime}(z)\left[\begin{array}{ccc}2 \lambda u_{0}^{\prime}(z)(1-\tilde{\beta}) & 0 & 1 \\ 0 & 0 & 0 \\ 1 & 0 & 0\end{array}\right]$.

Here the subscript 0 stands for the basic flow, $u_{0}^{\prime}(z)$ and $u_{0}^{\prime^{\prime}}(z)$ are the first and second derivatives of $u_{0}$ with respect to $z$, respectively. For the linear flow,

$u_{0}^{\prime}(1)=1, u_{0}^{\prime \prime}(1)=0$,

while for the return flow

$u_{0}^{\prime}(1)=1, u_{0}^{\prime \prime}(1)=\frac{3}{2}$.

It can be seen from (2.9c) that the main difference of the basic flow between Newtonian fluid and viscoelastic fluid is that the latter has a normal stress, which is caused by the elasticity of polymer.

\subsection{Perturbation equations}

Following the usual linear stability analysis, an infinitesimal normal-mode perturbation is imposed on the basic flow:

$(\mathbf{u}, T, P, \mathbf{Q})=\left(\mathbf{u}_{0}, T_{0}, P_{0}, \mathbf{Q}_{0}\right)+(\widehat{\mathbf{u}}, \widehat{T}, \widehat{p}, \widehat{\mathbf{Q}}) \exp [\sigma t+i(\alpha x+\beta y)]$,

$\widehat{\mathbf{u}}=(\widehat{u}, \widehat{v}, \widehat{w}), \widehat{\mathbf{Q}}=\left[\begin{array}{lll}\widehat{Q}_{11} & \widehat{Q}_{12} & \widehat{Q}_{13} \\ \widehat{Q}_{12} & \widehat{Q}_{22} & \widehat{Q}_{23} \\ \widehat{Q}_{13} & \widehat{Q}_{23} & \widehat{Q}_{33}\end{array}\right]$.
Here $\sigma=\sigma_{r}+i \sigma_{i}, \sigma_{r}$ and $\sigma_{i}$ are the growth rate and frequency of perturbation, respectively, $\alpha, \beta$ denote the wave number in the $x$ and $y$ directions respectively. The subscripts $1,2,3$ in $\widehat{Q}_{i j}$ stand for the $x, y, z$ directions, respectively. $k=\sqrt{\alpha^{2}+\beta^{2}}$ is the wave number and $\phi=\tan ^{-1}(\beta / \alpha)$ is the direction of wave propagation. Due to symmetry, we only need to consider the case of $\phi \in\left[0^{\circ}, 180^{\circ}\right]$. Hereafter, the variables without subscript 0 stand for the perturbation. It has been shown in [16] that, in thermocapillary convection, there is no Squire's theorem which ensures that the most dangerous mode is two-dimensional. The reason may due to the difference of the boundary condition between the thermocapillary convection (see (2.8)) and channel flows. Thus, we consider the three-dimensional disturbances directly.

The linearized equations of perturbation quantities are derived by substituting (2.12a)-(2.12b) into the governing equations as follows.

$$
\begin{aligned}
i \alpha \hat{u}+ & i \beta \hat{v}+\bar{w}^{\prime}=0 \\
R & {\left[\beta\left(\widehat{w} u_{0}^{\prime}+u_{0} i \alpha \widehat{u}\right)-\alpha\left(u_{0} i \alpha \widehat{v}\right)\right] } \\
& -\beta\left(i \alpha \widehat{Q}_{11}+i \beta \widehat{Q}_{12}+\widehat{Q}_{13}^{\prime}\right)+\alpha\left(i \alpha \widehat{Q}_{12}+i \beta \widehat{Q}_{22}+\widehat{Q}_{23}^{\prime}\right) \\
& =-\sigma R(\beta \widehat{u}-\alpha \widehat{v}),
\end{aligned}
$$

$$
\begin{aligned}
& R \alpha\left(D \widehat{w} \cdot u_{0}^{\prime}+\widehat{w}{u^{\prime}}_{0}+u_{0}^{\prime} \cdot i \alpha \widehat{u}+u_{0} i \alpha \widehat{u}^{\prime}\right)+R \beta\left(u_{0}^{\prime} \cdot i \alpha \widehat{v}+u_{0} i \alpha \widehat{v}^{\prime}\right) \\
& -\operatorname{Rik}^{2}\left(u_{0} i \alpha \widehat{w}\right) \\
& -\left(i \alpha^{2} \widehat{Q}_{11}^{\prime}+2 i \alpha \beta \widehat{Q}_{12}^{\prime}+\alpha \widehat{Q}^{\prime \prime}{ }_{13}+i \beta^{2} \widehat{Q}_{22}^{\prime}+\beta \widehat{Q}^{\prime \prime}{ }_{23}\right) \\
& +i k^{2}\left(i \alpha \widehat{Q}_{13}+i \beta \widehat{Q}_{23}+\widehat{Q}^{\prime}{ }_{33}\right) \\
& =-\sigma R\left(\alpha \widehat{u}^{\prime}+\beta \widehat{v}^{\prime}-i k^{2} \widehat{w}\right) \text {, }
\end{aligned}
$$

$M a\left(\widehat{u} \frac{\partial T_{0}}{\partial x}+\widehat{w} \frac{\partial T_{0}}{\partial z}+u_{0} i \alpha \widehat{T}\right)+\left(\alpha^{2}+\beta^{2}\right) \widehat{T}-\widehat{T}^{\prime \prime}=-\sigma M a \widehat{T}$,

$$
\begin{aligned}
(1 & \left.+\lambda\left(u_{0} i \alpha\right)\right) \widehat{Q}_{11}-\left(1+\eta\left(u_{0} i \alpha\right)\right) 2 i \alpha \widehat{u}-\lambda u_{0}^{\prime} 2 \widehat{Q}_{13} \\
& +\eta u_{0}^{\prime}\left(2 \widehat{u}^{\prime}+2 i \alpha \widehat{w}\right)+\eta u_{0}^{\prime} 2 \widehat{u}^{\prime} \\
& +\lambda\left(\widehat{w} u^{\prime \prime}{ }_{0} \cdot u_{0}^{\prime} 4(\lambda-\eta)-{u^{\prime}}_{0}\left(4(\lambda-\eta) u_{0}^{\prime} i \alpha \widehat{u}+2 \widehat{u}^{\prime}\right)\right) \\
& =\sigma\left(\eta 2 i \alpha \hat{u}-\lambda \widehat{Q}_{11}\right),
\end{aligned}
$$

$$
\begin{aligned}
(1 & \left.+\lambda\left(u_{0} i \alpha\right)\right) \widehat{Q}_{12}-\left(1+\eta\left(u_{0} i \alpha\right)\right)(i \alpha \widehat{v}+i \beta \widehat{u})-\lambda u_{0}^{\prime} \widehat{Q}_{23} \\
& +\eta u_{0}^{\prime}\left(i \beta \widehat{w}+\widehat{v}^{\prime}\right)+\eta u_{0}^{\prime} \widehat{v}^{\prime} \\
& +\lambda\left(-u_{0}^{\prime}\left(2(\lambda-\eta) u_{0}^{\prime} i \alpha \widehat{v}+\widehat{v}^{\prime}\right)\right)=\sigma\left(\eta(i \alpha \widehat{v}+i \beta \widehat{u})-\lambda \widehat{Q}_{12}\right),
\end{aligned}
$$

$$
\begin{aligned}
(1 & \left.+\lambda\left(u_{0} i \alpha\right)\right) \widehat{Q}_{13}-\left(1+\eta\left(u_{0} i \alpha\right)\right)\left(\widehat{u}^{\prime}+i \alpha \widehat{w}\right)-\lambda u_{0}^{\prime} \widehat{Q}_{33}+\eta u_{0}^{\prime}\left(2 \widehat{w}^{\prime}\right) \\
& -\eta\left(\widehat{w} u^{\prime \prime}{ }_{0}-u_{0}^{\prime}\left(\widehat{w}^{\prime}+i \alpha \bar{u}\right)\right) \\
& +\lambda\left(\widehat{w} u^{\prime \prime}{ }_{0}-u_{0}^{\prime}\left(2(\lambda-\eta) u_{0}^{\prime} i \alpha \widehat{w}+\widehat{w}^{\prime}+i \alpha \widehat{u}\right)\right) \\
& =\sigma\left(\eta\left(\widehat{u}^{\prime}+i \alpha \widehat{w}\right)-\lambda \widehat{Q}_{13}\right),
\end{aligned}
$$

$$
\begin{aligned}
& \left(1+\lambda\left(u_{0} i \alpha\right)\right) \widehat{Q}_{22}-\left(1+\eta\left(u_{0} i \alpha\right)\right)(2 i \beta \widehat{v})=\sigma\left(\eta(2 i \beta \widehat{v})-\lambda \widehat{Q}_{22}\right), \\
& \left(1+\lambda\left(u_{0} i \alpha\right)\right) \widehat{Q}_{23}-\left(1+\eta\left(u_{0} i \alpha\right)\right)\left(i \beta \widehat{w}+\widehat{v}^{\prime}\right)-\lambda u_{0}^{\prime} i \alpha \widehat{v}+\eta u_{0}^{\prime} i \alpha \widehat{v} \\
& =\sigma\left(\eta\left(i \beta \widehat{w}+\widehat{v}^{\prime}\right)-\lambda \widehat{Q}_{23}\right) \text {, } \\
& \left(1+\lambda\left(u_{0} i \alpha\right)\right) \widehat{Q}_{33}-\left(1+\eta\left(u_{0} i \alpha\right)\right)\left(2 \widehat{w}^{\prime}\right)-\lambda u_{0}^{\prime} 2 i \alpha \widehat{w}+\eta u_{0}^{\prime} 2 i \alpha \widehat{w} \\
& =\sigma\left(\eta\left(2 \widehat{w}^{\prime}\right)-\lambda \widehat{Q}_{33}\right) \text {. }
\end{aligned}
$$

Here, $\eta=\lambda \widetilde{\beta}$. The boundary conditions for the perturbation flow are set as follows.

$\widehat{u}=\widehat{v}=\widehat{w}=\frac{\partial \widehat{T}}{\partial z}=0, z=0$, 
$\widehat{Q}_{13}+\mathrm{i} \alpha \widehat{T}=0, \widehat{Q}_{23}+\mathrm{i} \beta \widehat{T}=0, \widehat{w}=0, \frac{\partial \widehat{T}}{\partial z}=0, z=1$.

We use Chebyshev-collocation method [27] to obtain the eigenvalue $\sigma . N_{c}$ Chebyshev-collocation points $z=\left(1-\cos \left(\frac{j \pi}{N_{c}+1}\right)\right) / 2, j=1 \sim N_{c}$ are set in the flow region for the Eqs. (2.13)-(2.22) while 2 points $z=0,1$ are set on the boundaries for the Eqs. (2.23)-(2.24). $\widehat{u}$ is expanded as

$\widehat{u}=\sum_{k=1}^{N_{c}+1} a_{k} H_{k-1}(\widehat{z})$.

Here $\widehat{z}=1-2 z, H_{k-1}(\hat{z})=\cos \left[(k-1) \cos ^{-1}(\widehat{z})\right]$ is the $(k-1)-$ th Chebyshev polynomial and $a_{k}$ is the coefficient. The cases of other perturbation quantities are similar. Then, the general eigenvalue problem can be derived in the form of $\mathbf{W g}=\sigma \mathbf{Z g}$, where $\mathbf{W}, \mathbf{Z}$ are two matrices, and $\mathbf{g}$ is the eigenvector. The eigenvalues can be obtained by using the QZ algorithm available in the Matlab-software package. In our work, the results are sufficiently accurate when $N_{c}=70 \sim 90$.

In order to validate our code, we provide a benchmark by solving the same problems for Newtonian fluid in previous works. The results of Newtonian fluid for the linear flow and return flow are computed by setting $\lambda=0$. The critical parameters are listed in Table 1 , which agree with the results in [22]. Here, $\psi=180^{\circ}-\phi$, and $c=\left|\sigma_{i}\right| / k$ is the wave speed.

\section{Numerical results}

Different from those in [21], here we focus on the flow at low Reynolds number and high elastic number. In the following, the Reynolds number $R=M a / P r=0.01$ in Figs. $2-6$ and 9, while $R=10^{-8} \sim 1$ in Figs. 7 and 8. It is found that unstable modes appear when the Weissenberg number exceeds a critical value.

\subsection{Elastic instability}

The variation of growth rate with wave number is displayed in Fig. 2 for a moderate Weissenberg number $(\lambda=1)$. There are unstable modes for both streamwise wave $\left(\phi=0^{\circ}\right)$ and oblique waves $\left(\phi \neq 0^{\circ}\right.$, $90^{\circ}$ ). The wave number of the unstable mode includes $O(k)=1$ and $O(k)=10$. Here, the most unstable mode is the oblique wave with the wave number $O(k)=10$.

It can be found in Fig. 2 that the variation of growth rate with $\phi$ is not monotonous. This is reasonable. The results of Newtonian fluid $[22,23]$ have shown that the preferred mode can either be the spanwise mode $\left(\phi=90^{\circ}\right)$, streamwise wave $\left(\phi=0^{\circ}\right)$ or oblique wave $\left(\phi \neq 0^{\circ}\right.$, $90^{\circ}$ ), which depends on the parameters. For the last case, the perturbation growth rate reaches its maximum in an oblique direction. We will solve the perturbation equations in the range of $\phi \in\left[0^{\circ}, 180^{\circ}\right]$ to find the preferred mode.

In order to check the convergence of our results, the eigenvalues are computed with different numbers of Chebyshev nodes and the results are displayed in Table 2. As the eigenvalues computed with $N_{c}=70 \sim 90$ are the same at least for the six decimal places, the numerical method is sufficiently accurate to find the instability.

In the following, we will derive the critical Weissenberg number $\lambda_{c}$, which is defined as the smallest Weissenberg number for the flow with a neutral mode $\sigma_{r}=0$. It is the condition for the onset of elastic

Table 1

The critical parameters for Newtonian fluid.

\begin{tabular}{lllll}
\hline$P r=0.1$ & $M a$ & $k$ & $\psi$ & $c$ \\
\hline Linear flow & 12.0 & 0.91 & $83.4^{\circ}$ & 0.046 \\
Return flow & 22.4 & 0.65 & $71.2^{\circ}$ & 0.070 \\
\hline
\end{tabular}

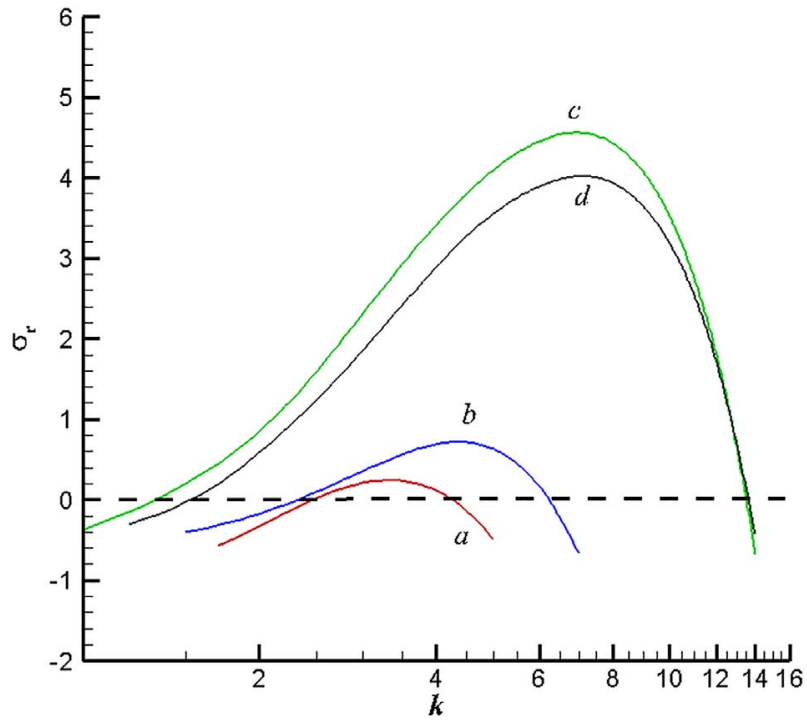

Fig. 2. The variation of growth rate with wave number for: (a) $\phi=0^{\circ}$ (streamwise wave); (b) $\phi=30^{\circ}$; (c) $\phi=67^{\circ}$; (d) $\phi=80^{\circ}$ in linear flow at $\lambda=1, M a=1, \operatorname{Pr}=100$.

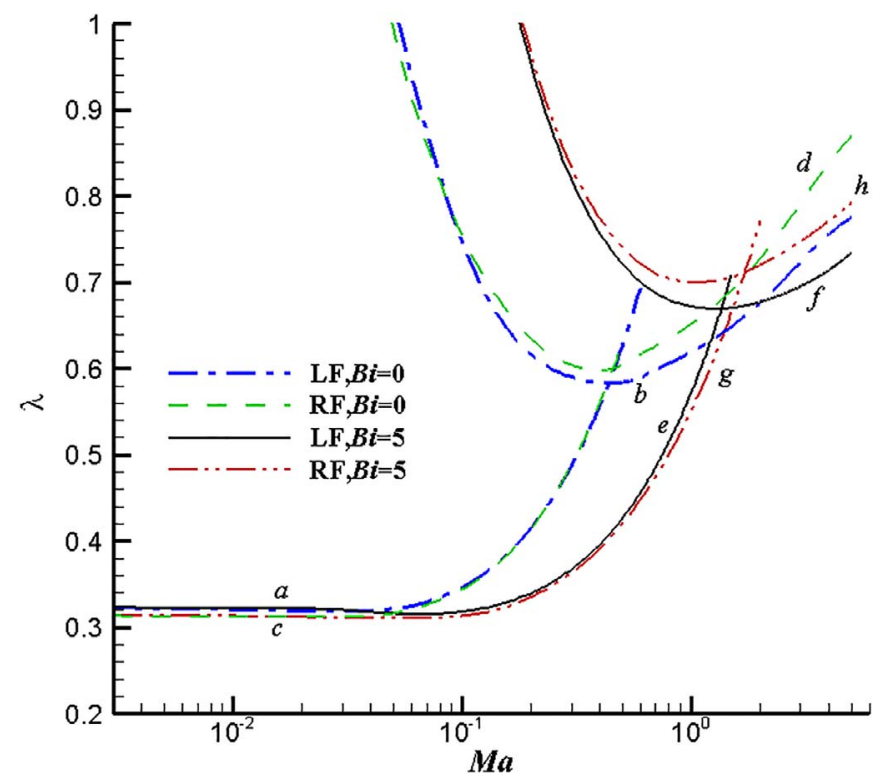

Fig. 3. The neutral curves ( $\lambda$ versus $M a$ ) for the linear flow (LF) and the return flow (RF). 1) The dash-dot line ( - - - denotes the linear flow at $B i=0$ : (a) streamwise wave, (b) oblique wave;

2) the dash line $(-\ldots=-a$ ) denotes the return flow at $B i=0$ : (c) streamwise wave, (d) oblique wave;

3) the solid line ( (f) oblique wave;

4) the dash-dot-dot line (_ u d u denotes the return flow at $B i=5:(\mathrm{g})$ streamwise wave, (h) oblique wave.

instability.

The neutral curves ( $\lambda$ versus $M a$ ) are displayed in Fig. 3. It can be seen that the flow state is susceptible to an elastic instability when Weissenberg number is in the order of $1 . \lambda_{c}$ changes little at small $M a$. Then it increases with $M a$ when $O(M a)=0.1$. The preferred mode changes from streamwise wave $\left(\phi=0^{\circ}\right)$ to oblique wave $\left(\phi \neq 0^{\circ}, 90^{\circ}\right)$ when $M a$ is large enough. $\lambda_{c}$ of the return flow is slightly smaller than that of the linear flow at small $M a$, while the case is reversed at large $M a$. The effect of Biot number is little at small $M a$. However, when $M a>0.1, \lambda_{c}$ is decreased by the increase of $B i$ in most cases. It suggests that $B i$ is destabilizing, which is opposite to the case of Newtonian fluid [22]. 


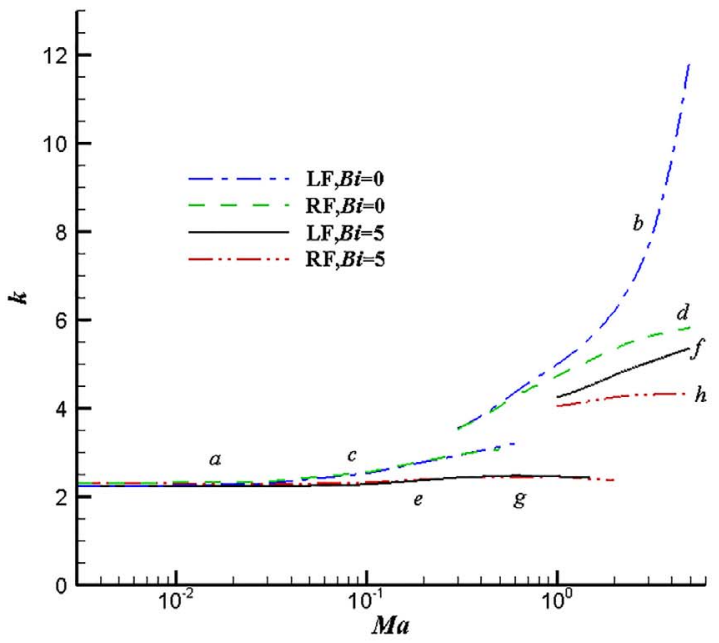

(a)

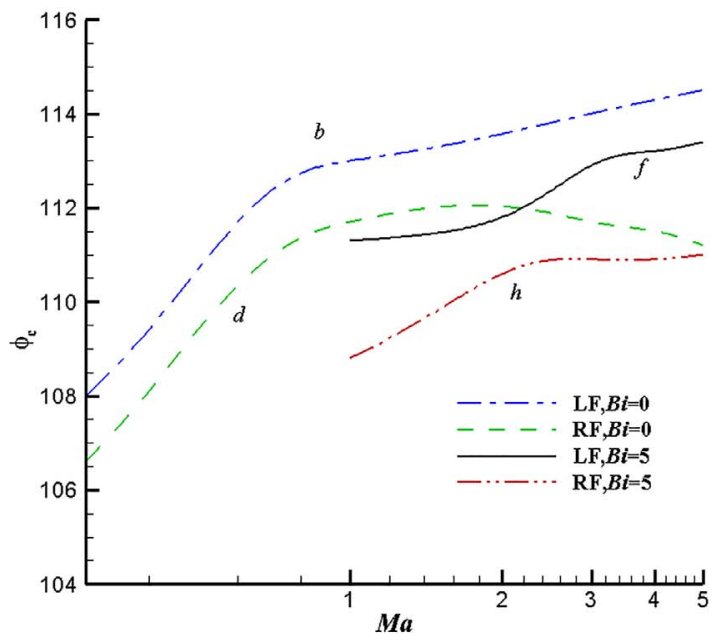

(b)

Fig. 4. The (a) wave number and (b) wave propagation angle corresponding to the mode in Fig. 3.

The wave number and wave propagation angle corresponding to the mode in Fig. 3 are displayed in Fig. 4. The variation of wave number with $M a$ is similar to that of $\lambda_{c}$. The wave number has $O(k)=10$ when $O(M a) \geq 1$. However, $k \approx 2.3$ when $M a \leq 0.1$. The oblique wave is always upstream and the propagation angle is close to $110^{\circ}$. We can see that $B i$ decreases the wave number and propagation angle at large $M a$. However, the differences are quantitative rather than qualitative.

The computation shows that when $M a<0.1$, the temperature perturbation is small. It can be inferred that the instability is not sensitive to the temperature field. However, when $M a$ is large, the temperature perturbation is obvious, and the Marangoni forces on the surface can dissipate the perturbation energy. Thus, the flow is stabilized by the temperature perturbation. This may be the reason why $\lambda_{c}$ increases with $M a$ in Fig. 3. As the heat flux through the surface decreases the temperature perturbation, the increase of $B i$ can lead to a decrease of $\lambda_{c}$. The instability at large $M a$ is affected by both the elasticity and thermocapillary effect. For simplicity, we will restrict our attention to the case at small $M a$, where the temperature perturbation is small.

An important feature of the elastic instability is the wave speed $c=\left|\sigma_{i}\right| / k$. The variation of wave speed with $M a$ and $\lambda$ is displayed in Fig. 5 at $k=2.26, P r=1$. It shows that $c \propto \frac{1}{\sqrt{M a}}, \frac{1}{\sqrt{\lambda}}$, which is similar to the property of elastic wave in plane shear flows for Burgers fluid [28]. The wave speed computed is dimensionless. We change the dimensionless wave speed into the dimensional quantity in order to elucidate its physical meaning. Let $c=\frac{1}{\sqrt{M a \cdot \lambda}} h$, where $h$ is a constant. Then, the dimensional wave speed has the form $C=c U=\frac{U}{\sqrt{M a \cdot \lambda}} h=\sqrt{\frac{G}{\rho}} \cdot \frac{h}{\sqrt{P r}}$, which is obviously an elastic wave speed.

It can be found that the wave speed in Fig. 5 is in the order of 100. In contrast, in [21], the wave speeds of oblique wave and streamwise wave are in the order of $0.1 \sim 1$, while the wave speed of spanwise stationary mode is zero. This suggests that the elastic instability in this paper is completely different from those in [21]. The reason is attributed to $\widetilde{\beta}$. The elastic wave in Fig. 5 decays rapidly with the increase of $\widetilde{\beta}$ as it has a high frequency. Therefore, the instability detected at $\widetilde{\beta}=0.001$ in this paper cannot be found in the case of $\widetilde{\beta}=0.1$ in [21].

We plot the preferred mode at $M a=0.01$ in Fig. 6 . The preferred mode is a two-dimensional streamwise wave. Its velocity and stress related to the spanwise direction are all zero: $v=0$, $Q_{12}=Q_{22}=Q_{23}=0$. The streamlines are the periodically arranged rolls in the streamwise direction. This is distinct from the streamwise wave in [21], whose velocity $u$ fluctuates several times in the vertical direction. It also differs from the spanwise mode and oblique wave in [21], which are both three-dimensional. To some extent, $w$ is symmetrical with respect to the line $z=0.55$. In contrast, the amplitude of $u$ at $z>0.55$ is obviously larger than that at $z<0.55$. This may due to their

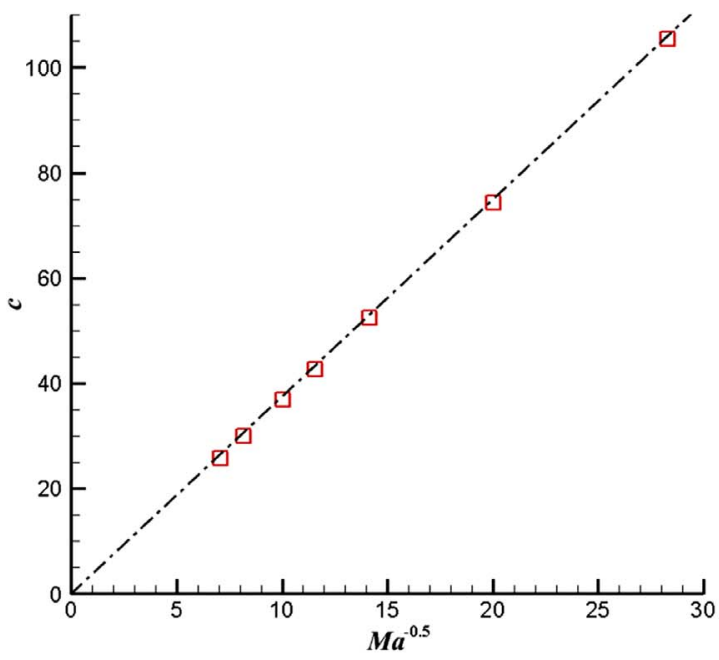

(a)

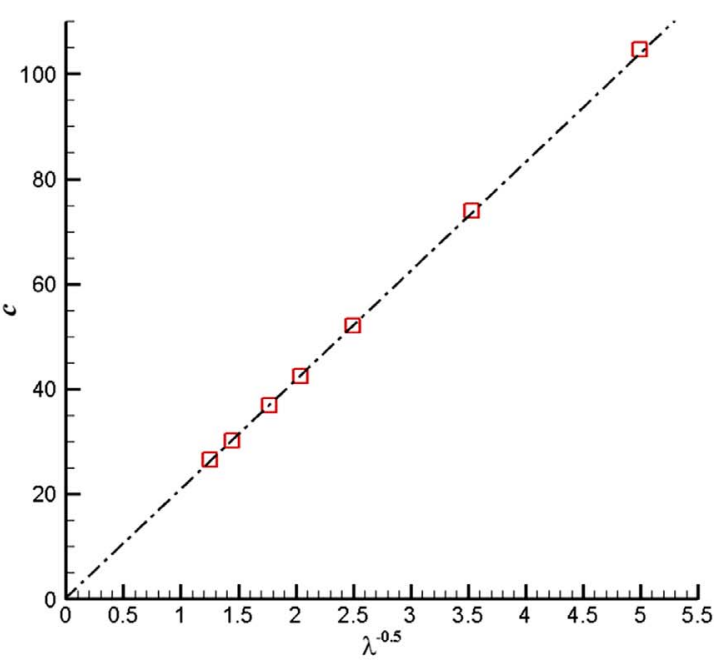

(b)

Fig. 5. The variation of wave speed $c$ at (a) $\lambda=0.321$ and (b) $M a=0.01$ for the streamwise wave in linear flow at $k=2.26, P r=1$. 


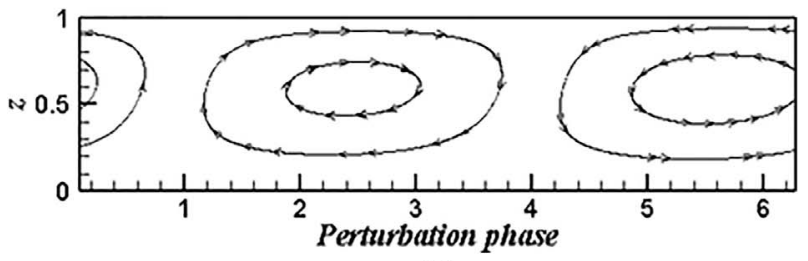

(a)

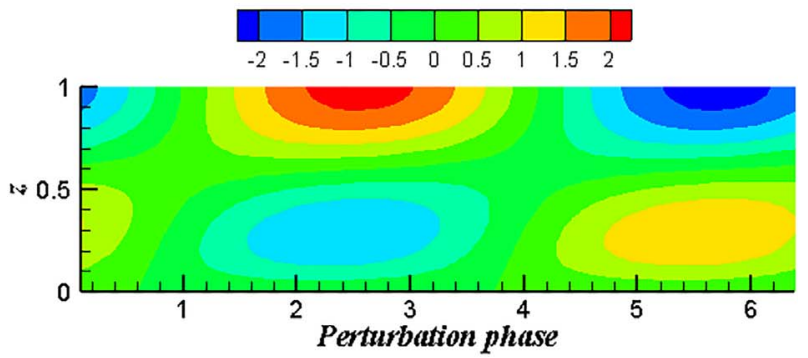

(b)

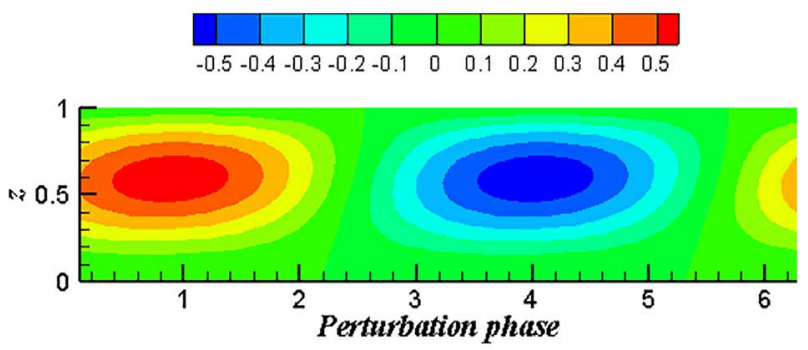

(c)

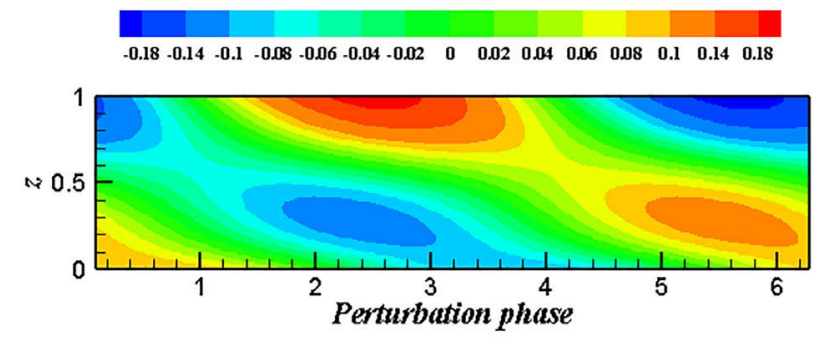

(d)

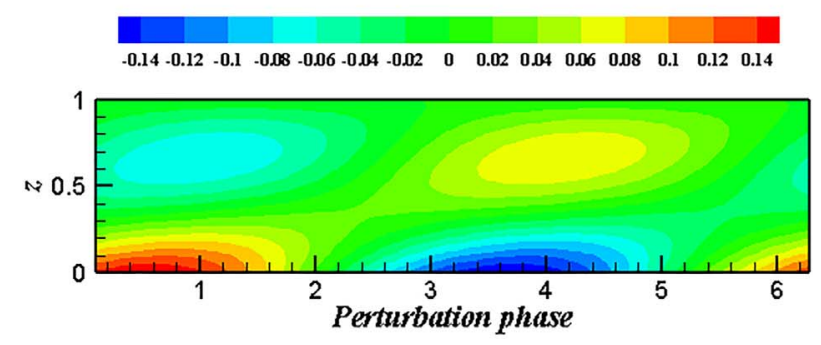

(e)

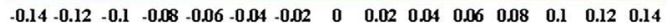

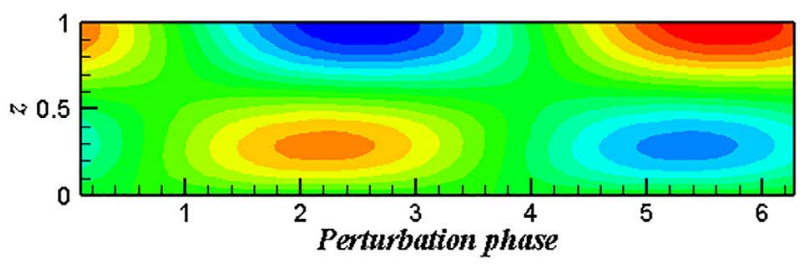

(f)
Fig. 6. The preferred mode for the linear flow at $M a=0.01, \lambda=0.321, k=2.26, \operatorname{Pr}=1$. (a) Stream lines; the distribution of the perturbation velocity: (b) $u$ and (c) $w$; the distribution of the perturbation stress: (d) $Q_{11}$, (e) $Q_{13}$ and (f) $Q_{33}$. Here, the preferred mode is a two-dimensional streamwise wave, whose velocity and stress related to the spanwise direction are all zero: $v=0, Q_{12}=Q_{22}=Q_{23}=0$.

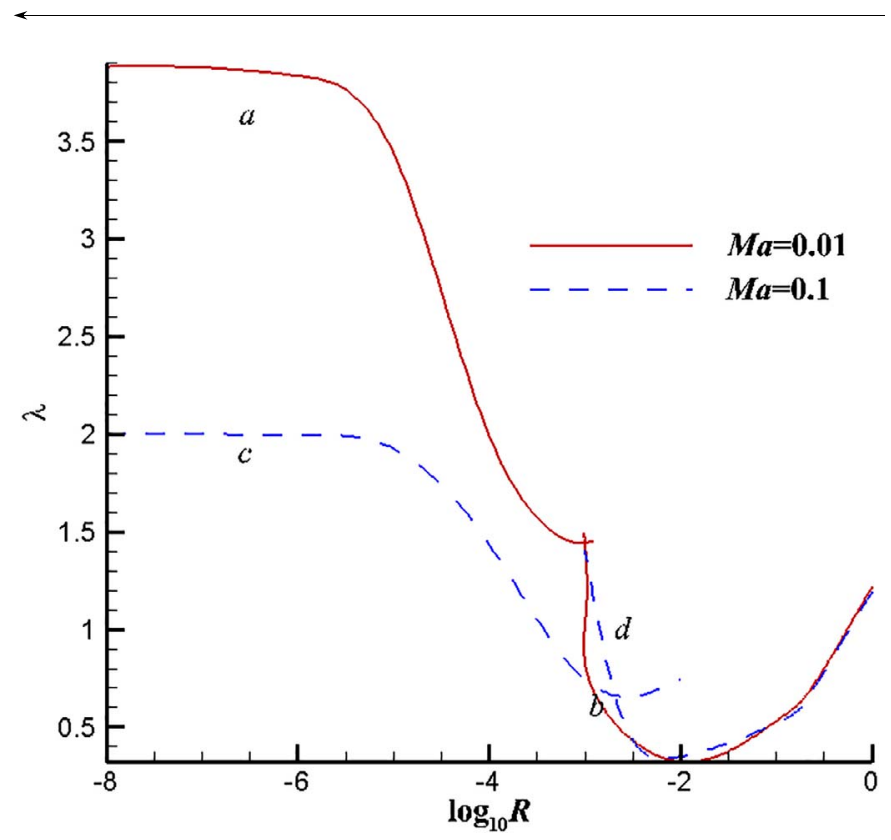

Fig. 7. The neutral curves $\left(\lambda\right.$ versus $\log _{10} R$ ) for the linear flow:

1) the solid line denotes the case at $M a=0.01$ : (a) oblique wave, (b) streamwise wave; 2) the dash line denotes the case at $M a=0.1$ : (c) oblique wave, (d) streamwise wave.

boundary conditions. For the former, $w=0$ at $z=0,1$, so there is a symmetry property for $w$. For the latter, there is no constraint on $u$ at $z=1$ while the boundary condition at $z=0$ is $u=0$, so the fluctuation near the surface is larger.

The amplitudes of $Q_{11}, Q_{13}$ and $Q_{33}$ have the same order. However, for the normal stress $\left(Q_{11}\right.$ and $\left.Q_{33}\right)$, the largest amplitude is on the surface, while for the shear stress $\left(Q_{13}\right)$, it is at the bottom. This is reasonable. The normal stress is caused by the stretching of the polymer chains, which is associated with the velocity gradient. As the surface has less constraint on the perturbation velocity, the normal stress can have the largest fluctuation on the surface. In contrast, the non-slip boundary condition at the wall is a large constraint on the perturbation velocity. The shear stress at the wall suppresses the growth of perturbation energy and leads to a strong dissipation (see Fig. 9). So we can expect a large shear stress at the wall.

As the largest amplitude of perturbation velocity is on the surface, the instability is closely related to the surface, although it is not an interfacial instability. To the best of our knowledge, this type of instability is not found in other shear flows of viscoelastic fluids, as they cannot induce a shear stress on the surface without imposing any constraint on the horizontal perturbation velocity.

The neutral curves $\left(\lambda\right.$ versus $\log _{10} R$ ) for the linear flow are displayed in Fig. 8 at $M a=0.01$ and $M a=0.1$. It can be seen that when $R \rightarrow 0$, the critical Weissenberg number $\lambda_{c}$ tends to a constant and the preferred mode is the oblique wave. $\lambda_{c}$ decreases with $M a$ significantly, which is contrast to the cases at $M a \geq 0.1$ in Fig. 3. When $R \approx 0.01, \lambda_{c}$ reaches its minimum and the preferred mode is the streamwise wave. When $R$ becomes larger, $\lambda_{c}$ increases with $R$ and is almost independent on $M a$.

The wave number, wave propagation angle and wave speed corresponding to the mode in Fig. 7 are displayed in Fig. 8. The wave number also tends to a constant when $R \leq 10^{-6}$. For the wave propagation angle and wave speed, the situations are similar. The variations of wave number and wave speed with $M a$ are small when $R \geq 10^{-2}$, while those 


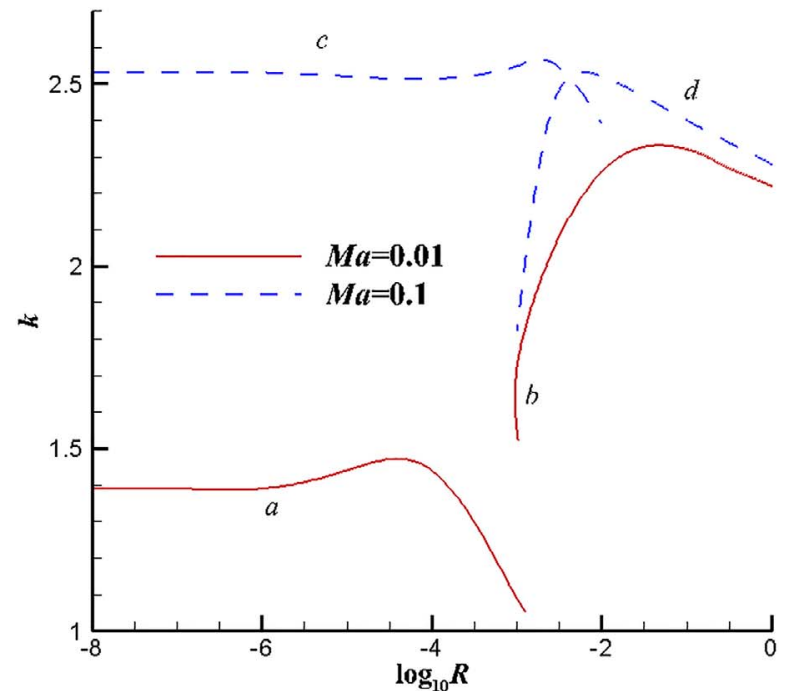

(a)

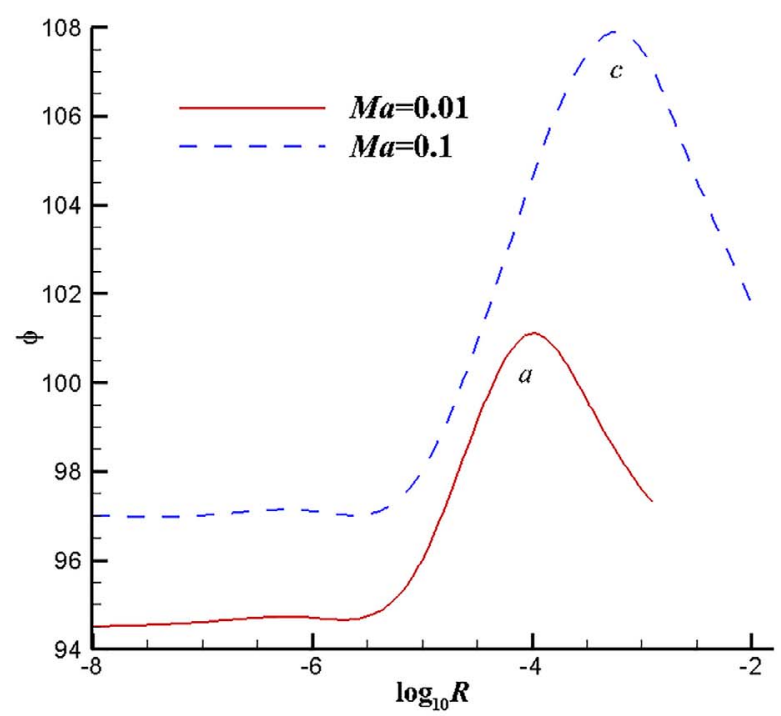

(b)

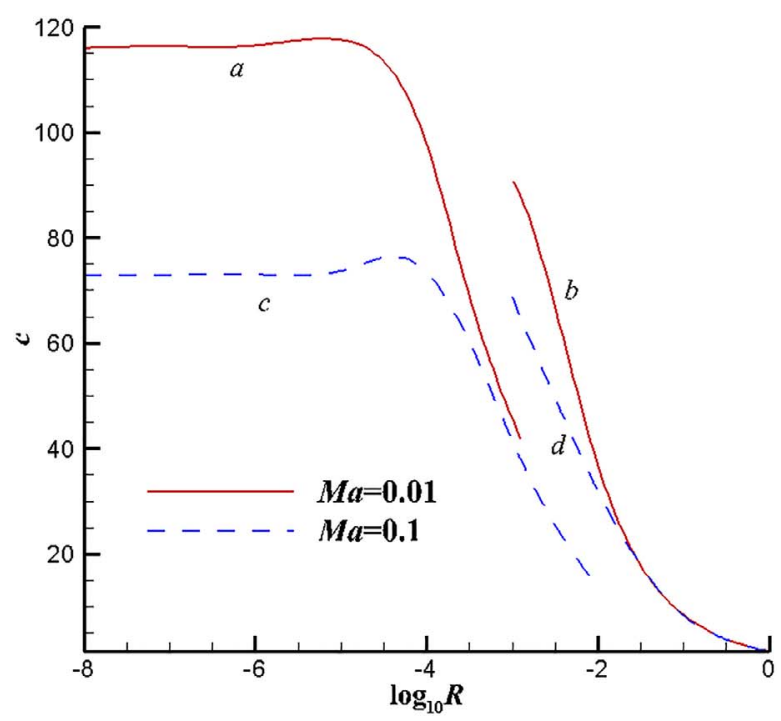

(c)

Fig. 8. The (a) wave number, (b) wave propagation angle and (c) wave speed corresponding to the mode in Fig. 7.
Table 2

The eigenvalues computed with different numbers of Chebyshev nodes at $\lambda=1, M a=1$, $\operatorname{Pr}=100, k=3$ in linear flow.

\begin{tabular}{lll}
\hline & $\phi=0^{\circ}$ & $\phi=67^{\circ}$ \\
\hline$N_{c}=70$ & $0.209010+50.386446 \mathrm{i}$ & $2.317419+30.662737 \mathrm{i}$ \\
$N_{c}=80$ & $0.209010+50.386446 \mathrm{i}$ & $2.317419+30.662737 \mathrm{i}$ \\
$N_{c}=90$ & $0.209010+50.386446 \mathrm{i}$ & $2.317419+30.662737 \mathrm{i}$ \\
\hline
\end{tabular}

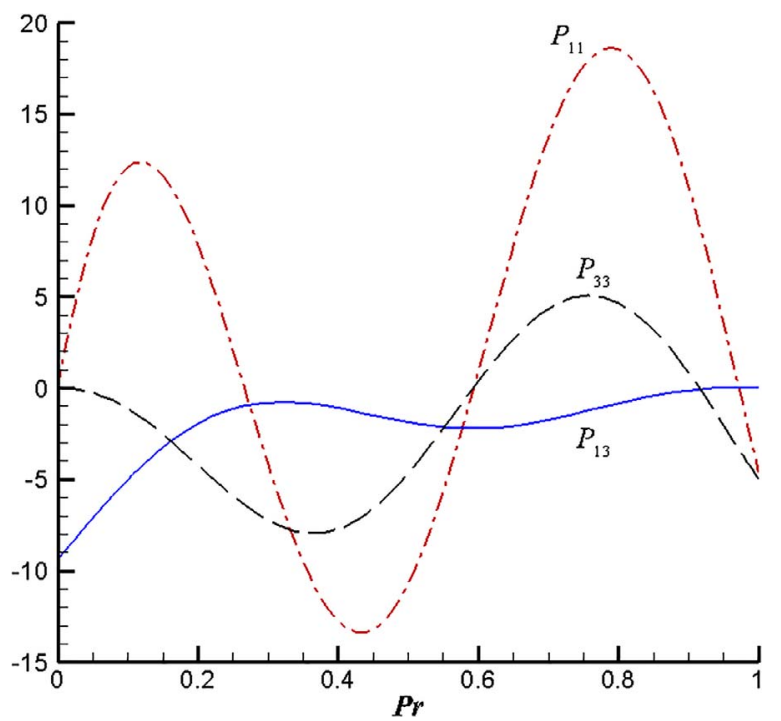

Fig. 9. The distribution of the work done by three components of stress for the linear flow at $M a=0.01, \lambda=0.321, k=2.26, \operatorname{Pr}=1$.

are obvious when $R \leq 10^{-4}$. The wave propagation angle $\phi$ increases with $M a$ for oblique waves, while $\phi=180^{\circ}$ for the streamwise waves in Fig. 7. The wave speed decreases with $R$ significantly when $R \geq 10^{-4}$.

The instability persists down to $R=0$, where the critical parameters are $\lambda_{c}=3.879, k=1.39, \phi=94.5^{\circ}$ at $M a=0.01$ and $\lambda_{c}=1.999, k=2.53$, $\phi=97^{\circ}$ at $M a=0.1$, respectively. This indicates that the inertia is not necessary for the instability.

\subsection{Energy analysis}

As shown in $[28,29]$, the rate of change of perturbation energy can be written as

$$
\begin{aligned}
\frac{\partial E_{k i n}}{\partial t} & =-\frac{1}{2 R} \int(\mathbf{Q}: \mathbf{S}) d^{3} r+\frac{1}{R} \int \mathbf{u} \cdot \mathbf{Q} \cdot \mathbf{n} d^{2} r-\int \mathbf{u} \cdot\left((\mathbf{u} \cdot \nabla) \mathbf{u}_{0}\right) d^{3} r \\
& =-N+M+I
\end{aligned}
$$

where $N$ is the work done by the perturbation stress, $M$ is the work done by Marangoni forces on the free surface, and $I$ is the energy comes from the basic flow, respectively. The perturbation is normalized as follows,

$\int \mathbf{u}^{2} d^{3} r=1$.

We find that $I$ is negligible at low Reynolds number, and $M$ is comparatively small. The perturbation energy mainly depends on $N$. As the perturbation stress fluctuates in the vertical direction (see Fig. 6), we plot the distribution of the work done by perturbation stress in vertical direction in Fig. 9. Here, the work done by three components of stress is defined as follows,

$P_{i j}=-\frac{1}{R} \int\left(Q_{i j} \cdot S_{i j}\right) \mathrm{d}^{2} r$.

There are strong fluctuations in the vertical direction for the work done by normal stress. The work done by the normal stress $Q_{11}$ in the 
region $z \in(0.65,0.95)$ makes a great contribution to the perturbation energy. There is a large dissipation of the shear stress $Q_{13}$ near the wall, while $P_{13}$ is close to zero near the surface $\left(Q_{13}\right.$ is nearly zero as the temperature perturbation is very small on the surface). It is conceivable that the wall can dissipate the perturbation energy, while the free surface has less constraint. This is the main reason why the channel flows with two solid boundaries are always linear stable at low Reynolds numbers while the instability appears in the thermocapillary liquid layer with a free surface, even though their velocity profiles of basic flow are similar (see Fig. 1).

For the streamwise wave in [21], the work done by perturbation stress also fluctuates in vertical direction, which seems similar to the case in Fig. 9. However, the former appears when Reynolds number is in the order of 100 . The effect of elasticity is deeply coupled with thermocapillary effect and the basic flow. So the instability of the former is not purely elastic.

\section{Discussion}

The instability in this paper is very different from that in [21]. First, we note that the elastic number $\varepsilon=\lambda / R$, which only depends on the properties of the fluid and the flow geometry [21]. It can be inferred from Fig. 3 that $\varepsilon$ is in the order of 100 , which is much larger than that in [21]. Therefore, the fluid in the present work has a higher elasticity. Meanwhile, in [21], most of the perturbation energy comes from the Marangoni forces when $\varepsilon$ is not very small $(O(\varepsilon) \geq 0.1)$. Thus, this instability cannot only be attributed to the elasticity. In contrast, we have shown in Section 3.2 that $I$ is negligible and $M$ is comparatively small. So the instability is purely elastic here. Another striking difference between the perturbation in this paper and that in [21] is the wave speed. The wave speed of the former is much larger than that of the latter. As the former has a high frequency, it decays rapidly with the increase of $\widetilde{\beta}$. Therefore, we cannot find it in [21].

In previous works, the elastic instability has been found by linear stability analysis in many flows with curved streamlines [30]. The critical condition for the onset of elastic instability is directly related to the curvature of the streamline. The complex coupling via normal stresses and curved streamlines is crucial for the instability mechanism. However, the thermocapillary convection in this paper gives an example of the elastic instability in the flow with straight streamlines. This suggests that the normal stress in parallel shear flow can also induce unstable modes.

It is worth noting that the plane Poiseuille and Couette flow are always linearly stable at low Reynolds numbers for Oldroyd-B fluid, although both of them also have the normal stress. In these channel flows, there can be large transient growths of small-amplitude disturbances, although they decay at long times. This phenomenon is related to the non-modal nature of the linear operator for the system. Hoda et al. [6,7] have studied the amplification of stochastic spatiotemporal body forces in channel flows of viscoelastic fluids. Their results indicate that the elasticity can amplify disturbances significantly even when inertial effects are weak. Furthermore, the transient growth [8], the non-modal amplification of stochastic [9] and deterministic [10] disturbances in inertialess plane Couette and Poiseuille flows of viscoelastic fluids have been demonstrated. These works suggest that the large transient growth could put the system into a regime where nonlinear interactions are no longer negligible. It seems that the nonmodal amplification [6-10] and nonlinearity [11] can lead to the elastic instability in channel flows, even the flows are linearly stable.

For thermocapillary convection, the linear stability analysis has successfully predicted the instability behaviours in the experiments of liquid bridge [31] and rectangular slot [32]. Therefore, we also use this method to examine the instability of viscoelastic thermocapillary convection. The instability detected by modal analysis in this paper differs from the non-modal amplification in channel flows. This difference can be attributed to the free surface of the thermocapillary convection.
Comparing with the solid boundary in channel flows, the free surface reduces the constraint on perturbation obviously, so the perturbation is more likely to grow at long times.

We can compare our results with some dimensional fluid parameters. Suppose the polymer liquid has the physical parameters as follows. The density $\rho$ is in the order of $10^{3} \mathrm{~kg} / \mathrm{m}^{3}$, the negative rate of change of surface tension with temperature $\gamma$ is in the order of $10^{-4} \mathrm{~N}$ / $\mathrm{m} \cdot \mathrm{K}$ [33], the viscosity $\mu$ is in the order of $0.4 \mathrm{~Pa} \cdot \mathrm{s}$ [34], the relaxation time of the fluid $\Gamma=\frac{\mu}{G}$ is in the order of 3 s, the Prandtl number $P r$ is in the order of 10. If we set the temperature gradient on the surface $b=3 \mathrm{~K} / \mathrm{cm}$ and the depth of the layer $d=1 \mathrm{~cm}$, then the Reynolds number $R$ is in the order of 0.01 , the Marangoni number $M a$ is in the order of 0.1 , the shear rate $\dot{\gamma}=U / d$ is in the order of $0.1 / \mathrm{s}$, and the Weissenberg number $\lambda=\frac{\mu}{G} \frac{U}{d}$ is in the order of 0.3 , which are close to the results in this paper.

\section{Conclusion}

In the present work, the stability of the thermocapillary convection at low Reynolds number and high elastic number $(O(\varepsilon)=100)$ is examined. The elastic instability is detected by linear stability analysis. The rheological property is modeled by the Oldroyd-B fluid, whose solvent viscosity is much less than the total viscosity.

The instability appears when Weissenberg number $\lambda$ is in the order of 1 . When $R \approx 0.01$, the critical Weissenberg number $\lambda_{c}$ reaches its minimum. The preferred mode includes the streamwise wave and oblique wave and the corresponding wave number varies from $O(k)=1$ to $O(k)=10$. The dimensional wave speed is proportional to $\sqrt{G / \rho}$, which is similar to an elastic wave speed. The difference between linear flow and return flow only appears when $M a$ is large enough. The heat flux through the surface destabilizes the flow, which is opposite to the case of Newtonian fluid. When $M a$ is small, the temperature perturbation is very small, and the instability is not sensitive to the temperature field. The instability differs from that in [21], as the wave speed of the former is much larger than that of the latter. When $R \rightarrow 0, \lambda_{c}$ tends to a constant. When $R \geq 0.01, \lambda_{c}$ increases with $R$ and is almost independent on Ma.

\section{Acknowledgments}

This work has been supported by the National Science Foundation of China (11402271 and 11532015) and K. C. Wong Magna Fund in Ningbo University. We also thank Pro. Davalos-Orozco for his helpful suggestions.

\section{References}

[1] R.G. Larson, Instabilities in viscoelastic flows, Rheol. Acta 31 (3) (1992) 213-263.

[2] R.M. Larson, S.G. Shaqfeh, S.J. Muller, A purely elastic instability in Taylor-Couette flow, J. Fluid Mech. 218 (1990) 573-600.

[3] P. Pakdel, G.H. McKinley, Elastic instability and curved streamlines, Phys. Rev. Lett. 77 (1996) 2459-2462.

[4] T.C. Ho, M.M. Denn, Stability of plane Poiseuiile flow of a highly elastic liquid, J. Non-Newtonian Fluid Mech. 3 (1977/1978) 179-195.

[5] M. Renardy, Y. Renardy, Linear stability of plane Couette flow of an upper convected Maxwell fluid, J. Non-Newtonian Fluid Mech. 22 (1986) 23-33.

[6] N. Hoda, M.R. Jovanovic, S. Kumar, Energy amplification in channel flows of viscoelastic fluids, J. Fluid Mech. 601 (2008) 407-424.

[7] N. Hoda, M.R. Jovanovic, S. Kumar, Frequency responses of streamwise-constant perturbations in channel flows of Oldroyd-B fluids, J. Fluid Mech. 625 (2009) $411-434$.

[8] M.R. Jovanovic, S. Kumar, Transient growth without inertia, Phys. Fluids 22 (2010) 023101.

[9] M.R. Jovanovic, S. Kumar, Nonmodal amplification of stochastic disturbances in strongly elastic channel flows, J. Non-Newtonian Fluid Mech. 166 (2011) 755-778.

[10] B.K. Lieu, M.R. Jovanovic, S. Kumar, Worst-case amplification of disturbances in inertialess Couette flow of viscoelastic fluids, J. Fluid Mech. 723 (2013) 232-263.

[11] L. Pan, A. Morozov, C. Wagner, P.E. Arratia, Nonlinear elastic instability in channel flows at low Reynolds numbers, Phys. Rev. Lett. 110 (17) (2013) 174502.

[12] T. Duffar (Ed.), Crystal Growth Processes Based on Capillarity: Czochralski, Floating Zone, Shaping and Crucible Techniques, John Wiley \& Sons, 2010. 
[13] G. Toussaint, H. Bodiguel, F. Doumenc, B. Guerrier, C. Allain, Experimental characterization of buoyancy-and surface tension-driven convection during the drying of a polymer solution, Int. J. Heat Mass Transf. 51 (17) (2008) 4228-4237.

[14] J.R. Felts, S. Somnath, R.H. Ewoldt, W.P. King, Nanometer-scale flow of molten polyethylene from a heated atomic force microscope tip, Nanotechnology 23 (21) (2012) 215301.

[15] J.P. Singer, Thermocapillary approaches to the deliberate patterning of polymers, J. Polym. Sci. (2017).

[16] O.A. Basaran, H. Gao, P.P. Bhat, Nonstandard inkjets, Annu. Rev. Fluid Mech. 45 (2013) 85-113.

[17] J.P. Downey, J.A. Pojman, Polymer Research in Microgravity: Polymerization and Processing, American Chemical Society, Washington, DC, 2001.

[18] A. Corbett, S. Kumar, Combined thermal and electrohydrodynamic patterning of thin liquid films, J. Eng. Math. 94 (2015) 81-96.

[19] P.N. Kaloni, J.X. Lou, On the convective stability of Oldroyd B fluid subject to a horizontal temperature gradient, ASME/JSME 2003 4th Joint Fluids Summer Engineering Conference, Honolulu, Hawaii, USA, 6-10, 2003, pp. 1601-1606.

[20] L.A. Davalos-Orozco, A.E. Chavez, Thermocapillary convection in a viscoelastic fluid layer under a horizontal temperature gradient, J. Appl. Polym. Sci. 49 (0) (1991) 141-153.

[21] K.X. Hu, M. He, Q.S. Chen, Instability of thermocapillary liquid layers for Oldroyd-B fluid, Phys. Fluids 28 (3) (2016) 033105.

[22] M.K. Smith, S.H. Davis, Instabilities of dynamic thermocapillary liquid layers. Part 1. Convective instabilities, J. Fluid Mech. 132 (1983) 119-144.

[23] R.J. Riley, G.P. Neitzel, Instability of thermocapillary-buoyancy convection in shallow layers. Part 1.Characterization of steady and oscillatory instabilities, J. Fluid Mech. 359 (1998) 143-164.

[24] Y.R. Li, N. Imaishi, T. Azami, T. Hibiya, Three-dimensional oscillatory flow in a thin annular pool of silicon melt, J. Cryst. Growth 260 (2004) 28-42.

[25] A.N. Morozov, W. van Saarloos, Subcritical finite-amplitude solutions for plane Couette flow of viscoelastic fluids, Phys. Rev. Lett. 95 (2005) 024501.

[26] D.D. Joseph, J.C. Saut, Change of type and loss of evolution in the flow of viscoelastic fluids, J. Non-Newtonian Fluid Mech. 20 (1986) 117-141.

[27] P.J. Schmid, D.S. Henningson, Stability and Transition in Shear Flows, Springer Science \& Business Media, 2012.

[28] K.X. Hu, J. Peng, K.Q. Zhu, The linear stability of plane Poiseuille flow of Burgers fluid at very low Reynolds numbers, J. Non-Newtonian Fluid Mech. 167-168 (2012) 87-94.

[29] M. Wanschura, V.M. Shevtsova, H.C. Kuhlmann, H.J. Rath, Convective instability mechanisms in thermocapillary liquid bridges, Phys. Fluids 7 (5) (1995) 912-925.

[30] E.S.G. Shaqfeh, Purely elastic instabilities in viscometric flows, Annu. Rev. Fluid Mech. 28 (1) (1996) 129-185.

[31] H.C. Kuhlmann, H.J. Rath, Hydrodynamic instabilities in cylindrical thermocapillary liquid bridges, J. Fluid Mech. 247 (1993) 247-274.

[32] C.L. Chan, C.F. Chen, Effect of gravity on the stability of thermocapillary convection in a horizontal fluid layer, J. Fluid Mech. 647 (2010) 91-103.

[33] G.T. Dee, B.B. Sauer, The surface tension of polymer liquids, Adv. Phys. 47 (2) (1998) 161-205.

[34] A. Groisman, V. Steinberg, Elastic turbulence in a polymer solution flow, Nature 405 (2000) 53-55. 